—REVIEW ARTICLE

Volume 8 Issue 32016

DOl: 10.5959/eimj.v8i3.457

ARTICLE INFO

Submitted: $25-06-2016$

Accepted: 11-08-2016

Online: 30-09-2016

\section{Medical Student Motivation and Well-being: A Systematic Review}

\author{
Mataroria P. Lyndon ${ }^{1,2}$, Thomas Masters ${ }^{3}$, Tzu-Chieh Yu ${ }^{4}$, Robert Shao ${ }^{5}$, \\ Hussain Alyami ${ }^{1,2}$, Marcus Henning ${ }^{4}$, Andrew G. Hill ${ }^{2}$ \\ ${ }^{1}$ Ko Awatea, Counties Manukau District Health Board, New Zealand \\ ${ }^{2}$ South Auckland Clinical Campus, The University of Auckland, New \\ Zealand \\ ${ }^{3}$ University of Otago, New Zealand \\ ${ }^{4}$ Centre for Medical \& Health Sciences Education, The University of \\ Auckland, New Zealand
}

${ }^{5}$ Counties Manukau District Health Board, New Zealand

To cite this article: Lyndon MP, Masters T, Yu T-C, Shao R, Alyami H, Henning M et al. Medical student motivation and well-being: a systematic review. Education in Medicine Journal. 2016;8(3): 11-20. http://dx.doi.org/10.5959/eimj.v8i3.457

To link to this article: http://dx.doi.org/10.5959/eimj.v8i3.457

\title{
ABSTRACT
}

Introduction: Research directly applying theories of motivation to the well-being of medical students remains scarce, likely heterogeneous, and widely dispersed. A systematic review was therefore conducted to summarise and critically appraise current evidence of the relationship between medical students' motivation and their well-being. Methods: In December 2013, a systematic literature search of four electronic databases (Medline, EMBASE, Psychinfo, ERIC) was completed. Articles focusing on both medical student motivation and well-being were included. Selection was based on strict eligibility criteria after examining full-texts. Results: From 2,204 potential titles, 26 full text articles were assessed for eligibility, and nine studies met review inclusion criteria. Studies demonstrated significant associations between motivation (including intrinsic, extrinsic, amotivation, self-efficacy, and self-regulation) and well-being (including quality of life, stress, anxiety, or exhaustion), which was moderated by demographic and curriculum variables. Furthermore, a relationship may exist between, motivation, well-being, and academic achievement. There was significant heterogeneity in the study outcomes, and risks of bias were common in study methodologies. Conclusions: There is evidence to suggest a relationship between medical student motivation and well-being, and academic achievement. However, heterogeneity and risks of bias were common in study methodologies. Further research is needed to explain demographic differences, and how curricula can promote optimal motivation and well-being for all medical students.

Keywords: Motivation, Well-being, Quality of life, Medical students, Academic achievement 


\section{Introduction}

To effectively develop the knowledge, skills and competencies expected of clinicians, there is evidence to suggest that medical students need to maintain functional levels of personal health and well-being (1). However, the intensity of the medical training process may have unintended negative consequences $(2,3)$. Medical students are more likely to manifest symptoms of depression and anxiety when compared with non-medical peers and the general population. These findings are consistent with studies that have shown that medical students are vulnerable to psychological distress such as stress, burnout and poor mental quality of life (QOL) that can impact motivation for learning and academic achievement $(2,4)$.

These associations have been observed in the medical education literature. Findings from Kusurkar et al. (5) suggested that medical students who are more intrinsically motivated experience less exhaustion from study and achieve higher grade point averages (GPAs) when compared with students who are more extrinsically motivated or who have low motivation (6). Similarly, studies of medical student motivation and well-being have also shown significant correlations between the type of motivation, psychological health, and academic achievement $(4,7)$.

However, despite these findings, studies of the relationship between motivation and well-being in medical education remain scarce, dispersed and seemingly heterogeneous. Therefore, a systematic review was conducted to summarise and critically appraise the current evidence base for the relationship between motivation and well-being among medical students as a platform for future research. To address this aim, the following research questions are addressed in this review:

1. How have researchers measured motivation and well-being among medical students?
2. What factors influence motivation and well-being among medical students?

3. Which specific motivational orientations are associated with enhancing or adversely affecting well-being, and vice versa?

4. What are the effects of motivation and well-being on medical student academic achievement?

5. What are the limitations of current research in this area?

\section{Method}

In December 2013, a literature search was conducted of four electronic databases: MEDLINE, EMBASE, PsycINFO and ERIC. The search terms that were used were grouped into three key concepts: "motivation", "well-being" and "medical students".

Theories of motivation and their associated concepts were incorporated into the search terms for this review and included motivation, self-efficacy, extrinsic and intrinsic motivation, self-determination theory, social cognitive theory, goal theory, expectancy-value theory, achievement motivation, drive, stimulus, impetus, interest, and amotivation.

Similarly, as the term "well-being" is a multidimensional construct, a variety of keywords were used, including quality of life, mental health, depression, anxiety, distress, happiness and burnout. Search terms for "medical students" included both medical students and medical education. The search was limited to articles published in English. A bibliographical management program (EndNote X6, Thomson Reuters, New York) was used to create a search library and the references for the research articles were retrieved for examination. The following guidelines were adhered to with regard to the search strategy:

1. All identified abstracts were reviewed and full articles were retrieved by the reviewers when it was clear that they 
contained discussion about medical student motivation and well-being.

2. The researcher met with an independent reviewer to select articles for critical appraisal and review. Any discrepancies in opinion were identified and final agreement was reached after face-to-face discussion between the researcher and reviewer.

3. A hand search of the reference lists from these articles was conducted to determine further articles that could be considered for this review.

4. The reviewers met to determine and select articles for critical appraisal and review based on explicit inclusion and exclusion criteria.

5. Each selected article for review was analysed and data were collected independently by the researcher and the independent reviewer.

\section{Inclusion Criteria}

1. All study participants were medical students (individuals enrolled in a school of medicine).

2. Study measures encompassed both medical student motivation, and well-being (including QOL, stress, depression, anxiety, distress, happiness and burnout).

\section{Exclusion Criteria}

1. Full text of article not published in English.
2. Study results duplicated in separate earlier publications.

3. Brief descriptive, commentary or review articles.

4. Studies published prior to 1990 .

\section{Critical Appraisal}

Each selected article was analysed independently by at least two reviewers and data extracted using a critical appraisal instrument (Table 1) (8). To integrate the extracted data, a thematic analysis was performed. The most relevant themes from each article were identified and grouped according to the research questions within this review.

To assess the quality of the studies included in the review, the reviewers considered risks of bias within each study, including methods of recruitment, assessment of outcomes and reporting of outcomes. This framework was not constructed to exclude methodologically weak studies, but rather as an aid for reviewers to draw conclusions about the validity and reliability of the evidence presented in each study. Any discrepancies between the reviewer selections were identified and consensus was reached after face-to-face discussion.

\section{Results}

The search strategy yielded 2,731 articles. A total of 26 articles were identified by two independent reviewers as potentially relevant

Table 1: Data extraction and critical appraisal instrument

\begin{tabular}{ll}
\multicolumn{1}{c}{ Study introduction } & \multicolumn{1}{c}{ Research objectives and rationale } \\
\hline Study context & $\begin{array}{l}\text { Study setting, participant characteristics, description of the medical } \\
\text { programme }\end{array}$ \\
Study methods & $\begin{array}{l}\text { Study design, participant recruitment, study duration and } \\
\text { follow-up, description of measures of motivation and well-being, } \\
\text { assessment of study outcomes, methods of statistical analysis }\end{array}$ \\
Study results & Main study findings \\
Study conclusions & Future implications and new insights \\
Risks of bias within each study & Selection bias, outcome bias \\
\hline
\end{tabular}


based on the inclusion and exclusion criteria and after screening the titles and online abstracts of the initial 2,204 articles (after removal of duplicates). These were obtained as full texts for review. An additional five papers from a hand search of key journals and from reference lists were also included. Of the 26 articles identified, the inclusion and exclusion criteria were used to select a total of nine articles for critical appraisal (Figure 1). Of these studies, one was qualitative and eight were quantitative.

\section{Study Question 1: How Have Researchers Measured Motivation and Well-Being among Medical Students?}

The most common method of measuring motivation was self-reported questionnaires adapted from pre-existing tools. An adapted version of the Academic Motivation Scale (AMS) by Vallerand et al. (10) was used in three studies $(5,7,11)$. Three studies used subscales of the Motivated Strategies for Learning Questionnaire (MSLQ) (1, 4).
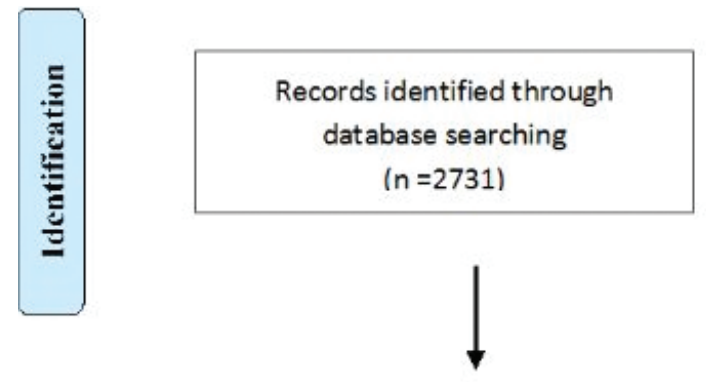

Records after duplicates removed

$(n=2204)$
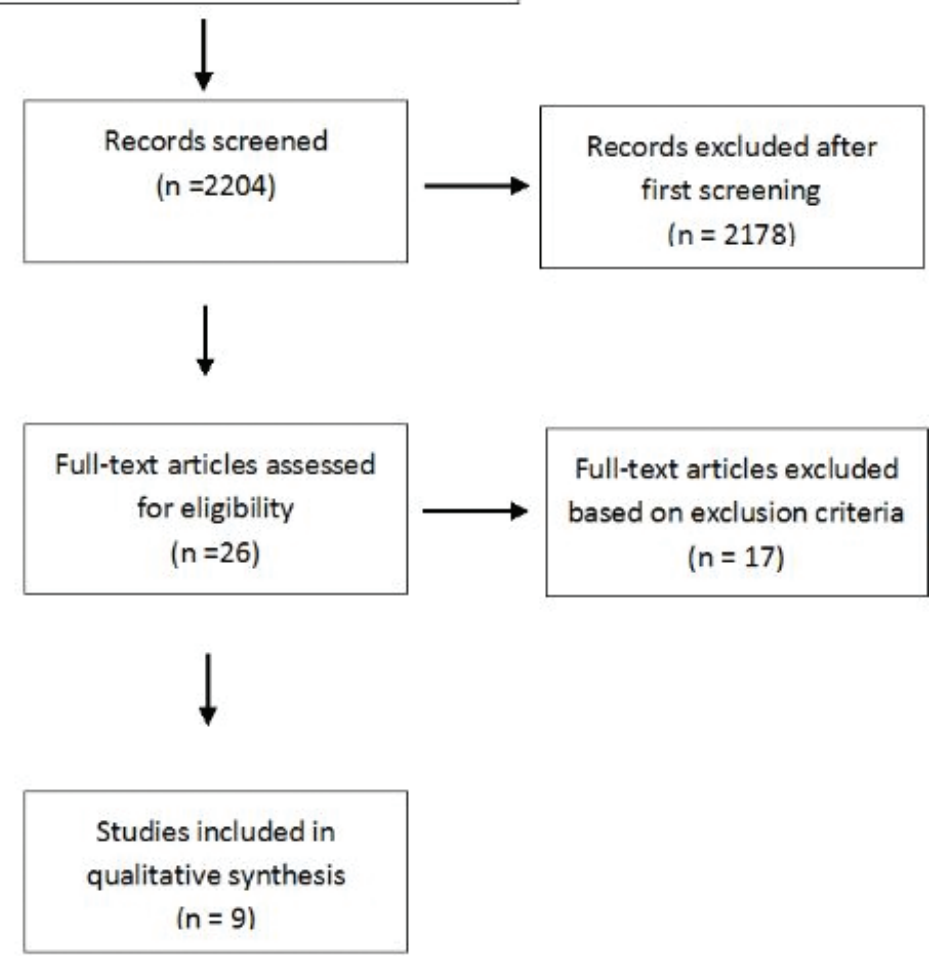

Studies included in
Additional records identified through other sources $(n=5)$

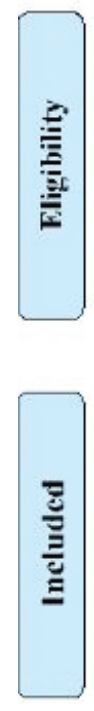

Figure 1: PRISMA statement of search results (9). 
Artino et al. (12) also used subscales from their previously published studies, which measured task value and self-efficacy (12). The Achievement Motivation Scale was used by Srivastava et al. (13).

Self-reported questionnaires were also the most common method of measurement of well-being. These questionnaires were created or adapted by investigators in order to measure stress, anxiety, exhaustion, boredom, depression, enjoyment and QOL. The WHOQOL-BREF measuring dimensions of QOL was used in three studies (1, 4). The Beck Depression Inventory, which evaluates the presence and severity of various depressive symptoms, was used in two studies $(7,11)$. Other tools used to measure well-being included the Achievement Emotions Questionnaire (AEQ) (12), the Social Adjustment ScaleSelf-Report (SAR-SR) (11), the Beck Anxiety Inventory (BAI) (11), the Maslach Burnout Inventory-Student Survey (MBISS) (5), the Medical Stress Scale (MSS) (7), the Hamilton Rating Scale for Depression (HAM-D-17) (7), and the Institute for Personality and Ability Testing (IPAT) (13).

\section{Study Question 2: What Factors Influence Motivation and Well-Being among Medical Students?}

\section{Phase of Curriculum}

Four studies reported associations between the phases of the medical curriculum and motivation and well-being. Findings from Park et al. (7) showed that students in their first year of medical school experience higher levels of stress compared with students in their second and third year of medical school. Similarly, Del-Ben et al. (11) reported high levels of anxiety among first-year medical students that increased during an academic year; and a decline in the quality and time available for leisure activities.

In the same study by Del-Ben et al. (11), there was also a significant reduction in Intrinsic Motivation (IM) scores, identified
Extrinsic Motivation (EM) scores and introjected EM scores during the academic year. However, there were no significant changes in external regulation and Amotivation (AM) scores.

Srivastava et al. (13) assessed changes in motivation and well-being at three points over a three-year period. The findings from this study showed a significant decrease in achievement motivation and an increase in physiological complaints by students. However, there were no differences in anxiety scores and psychological adjustment over the same period.

Henning et al. (1) conducted focus groups to explore factors that influence motivation to learn and QOL among medical students during the clinical phase of a medical curriculum. This study identified that anxiety and uncertainty in the clinical setting, transport issues to access clinical attachments, sleep deprivation and isolation from peer groups can negatively influence motivation to learn and QOL. However, positive experiences that provided insights into future clinical practice had a positive influence on motivation to learn and QOL.

\section{Demographic Characteristics}

An interaction between the demographic characteristics of students and their motivation and well-being was reported in two studies. Henning et al. (14) examined the relationship between QOL, motivation to learn and demographic variables, including age, gender and ethnicity. In this study, Asian medical students were compared with NZ European medical students. The findings showed that Asian medical students scored lower than NZ European students on measures of social QOL; however, there were no significant differences in self-efficacy, task value and intrinsic goal motivation. In addition, no gender or age effects were noted. Similarly, a study by Artino et al. (15) demonstrated no gender effect in relation to task value, and self-efficacy, and course-related anxiety and worry. 
In contrast, Kusurkar et al. (5) reported a gender effect on motivation and exhaustion among medical students. A higher proportion of male students were in the status-motivated profile (low intrinsic, high extrinsic controlled motivation), whereas a higher proportion of female students were in the interest-motivated profile (high intrinsic, low extrinsic controlled motivation). Female students also scored higher on measures of study-related exhaustion compared with male students.

\section{Study Question 3: Which Specific Motivational Orientations Are Associated with Enhancing or Adversely Affecting Well-Being, and Vice Versa?}

Five studies investigated the relationship between motivation and well-being. Henning et al. (4) noted correlations between self-efficacy, intrinsic value and self-regulation in relation to the physical, environmental, psychological and social QOL of medical students in their clinical years of training. A further study by Henning et al. (14) found similar correlations among Asian medical students.

Kusurkar et al. (5) identified a relationship between motivation and exhaustion. Results of this study showed that medical students with both high IM and low extrinsic controlled motivation, had significantly lower exhaustion from study than students with low intrinsic/high extrinsic controlled motivation or low intrinsic/low extrinsic controlled motivation.

Both Park et al. (7) and Artino et al. (12) identified a relationship between stress and anxiety and motivation. In the study by Park et al., students with higher stress scores scored highest on measures of AM and extrinsic identified regulation, and lowest on measures of IM when compared with students with lower stress scores. In the study by Artino et al., students with higher anxiety scores reported lower levels of selfefficacy motivation in comparison with students with lower anxiety scores.

\section{Study Question 4: What Are The Effects of Motivation and Well-Being on Medical Student Academic Achievement?}

Five studies investigated the relationship between motivation, well-being and academic achievement. Artino et al. (12) conducted a longitudinal study of secondyear medical students' motivational beliefs, achievement emotions and academic performance. This study reported that task value and self-efficacy were positively correlated with student enjoyment and course examination grade, whereas anxiety was negatively correlated with course examination grade.

Similarly, a study by Park et al. (7) showed a negative association between stress and academic achievement. In this study, students with high stress scores scored lower on their GPA than students with low stress scores.

Henning et al. (4) investigated the associations between QOL, motivation and academic achievement. The findings of this study suggested a positive correlation between students' academic achievement on written grades and measures of their QOL and motivation. However, there were no associations found between motivation and QOL with achievement in the clinical setting.

Kusurkar et al. (5) compared motivational profiles of medical students and showed that medical students with high IM had significantly deeper study strategies, higher self-study hours and higher GPAs than students with high EM or low motivation.

Artino et al. (15) investigated the effects of academic achievement on motivation and well-being. This study's findings suggested that low-achieving students had lower task value and self-efficacy motivation, and greater anxiety, frustration and boredom, when compared with high-achieving students. 
In contrast to the previous studies, Del-Ben et al. (11) found that academic achievement did not correlate significantly with any measure of academic motivation or wellbeing in a medical student population. The study indicated that increased anxiety, decreased academic motivation and a maladjusted leisure/social life had no significant correlations with examination grades among first-year medical students.

\section{Study Question 5: What Are The Limitations of Current Research in This Area?}

\section{Study Design}

The nine studies selected for this review were all observational studies; five of the studies used a single group study design $(4,7,11-13)$ and four used a comparison group for assessment of outcomes (1, 5, $14,15)$. There was a large variety in study sample sizes: the smallest study contained 19 participants (1) and the largest study had 844 (5). Three studies did not report response rate $(1,7,13)$.

\section{Participant Sampling and Selection Bias}

Participant sampling was not reported in studies by Srivastava et al. (13) and Park et al. (7). A response bias was a potential bias in some studies, especially those with lower response rates, such as the study by Kusurkar et al. (5) with a response rate of $44 \%$. No studies reported any prerequisite selection criteria, aside from participants needing to be enrolled in a medical school.

Attrition bias was potentially present in longitudinal studies, as differences between participants who dropped out of the studies or were lost to follow-up were not reported, except in the study by Del-Ben et al. (11). In studies utilising comparison groups, baseline differences in known confounders such as age and gender were controlled for during statistical analysis and the results reported appropriately.

\section{Outcome Measurement Bias}

All but one study (13) commented on the reliability and validity of the tools used to measure student motivation and wellbeing. In addition, only Artino et al. (12) commented on reliability of the measures of academic achievement. In the study by Henning et al. (4), academic achievement was not measured by actual grades of the participating students but instead was measured using estimations of grades reported by the participants themselves. Therefore, a social desirability bias may be present in this study (16).

\section{Discussion}

The findings of this review indicate a relationship between motivation and well-being that can be influenced by demographic and curriculum factors. Furthermore, this review indicates that motivation and well-being can influence academic achievement. However, there was significant heterogeneity in study outcomes, and risks of bias were common in study methodologies.

These findings have implications for student equity and learning. From an equity perspective, medical curricula and training should aim to produce equitable outcomes among medical students. However, the results of this review suggest differences in the experience of psychological distress among female students and ethnic minorities. Similar findings have been observed by Dyrbye et al. (3, 17), who previously reported higher levels of psychological distress among female medical students and ethnic minorities. However, not all studies as reported in this review showed similar findings. Furthermore, only one study in this review explored ethnic group differences in motivation and wellbeing, showing differences in QOL among Asian medical students (14). Further research is needed to explain why motivation and well-being may differ by gender and ethnicity with the view to gaining insight 
into potential sociocultural influences and differences in the educational experiences among these students during medical school.

From an academic learning perspective, there is evidence of medical student motivation and well-being being undervalued in the development of medical curricula $(2,18)$. However, consideration of how curricula affect motivation and wellbeing is important, as the findings of this review suggest changes in motivation and increasing psychological distress as students' progress through a curriculum. Therefore, considerations of how curricula can promote optimal motivation and well-being should be considered as part of a future research agenda and any curriculum reforms.

Of the studies that investigated associations between motivation and well-being, and academic achievement, the evidence presented suggests that there may be an association; however, the low magnitude of the correlations makes it difficult to ascertain the relative level of educational impact. A future research agenda should consider effect sizes and the significance of these associations longitudinally. A qualitative approach may also assist in understanding the underlying mechanisms of why motivation, well-being and academic achievement are associated.

Future research could also consider motivation and well-being not only in the context of medical education, but also studies that exist in other fields. For example, Sheldon \& Krieger (19) conducted a study among law students which showed a decline in motivation and well-being over time - similar to medical students. The learnings from this study including the influence of teacher behaviour on student motivation and well-being which may have relevance for medical educators. Thus, literature from other fields could incorporated into future systematic reviews of motivation and well-being.

Given the importance of motivation and well-being for medical education, the number of studies published in this area are scarce and have significant limitations. Firstly, there was considerable heterogeneity in the measures used to determine motivation and well-being among medical students. This diversity is in part a reflection of the range of theories of motivation that are currently present in the literature, including Self-Determination Theory (SDT), from which the AMS originates, as well as the MSLQ, which was developed using a social cognitive view of motivation $(10,20,21)$. Measures of well-being also varied significantly: author selected to measure levels of depression, anxiety, exhaustion, boredom, stress, enjoyment and QOL. This is a limitation when trying to synthesise and compare results from different studies, and as a result, a metaanalysis of study results was unable to be performed.

These limitations also extend to risks of bias throughout the reviewed studies. These risks were heightened by a lack of description of participant selection methods and low participant response rates. The significance of social desirability bias must also be considered a result of self-reporting within many of the reviewed studies. Social desirability bias has been suggested to influence self-reporting of psychological well-being, burnout and academic motivation among students in higher education (22-24). Researchers using selfreport questionnaires should consider the effect of socially desirable responding on the validity of their research.

\section{Conclusion}

This systematic review has summarised and critically appraised current evidence of the relationship between medical student motivation and well-being, the factors that influence motivation and well-being, and their effects on student learning outcomes. There is evidence to suggest a relationship between medical student motivation and well-being, and academic achievement. 
However, heterogeneity and risks of bias were common in study methodologies. Further research is needed to explain demographic differences, and how curricula can promote optimal motivation and wellbeing for all medical students.

\section{References}

1. Henning MA, Krägeloh C, Hawken S, Zhao Y, Doherty I. Quality of life and motivation to learn: a study of medical students. Issues in Educational Research. 2010;20(3):24456.

2. Dyrbye LN, Thomas MR, Shanafelt TD. Medical student distress: causes, consequences, and proposed solutions. Mayo Clinic Proc. 2005;80(12):1613-22. http://dx. doi.org/10.4065/80.12.1613.

3. Dyrbye LN, Thomas MR, Shanafelt TD. Systematic review of depression, anxiety, and other indicators of psychological distress among U.S. and Canadian medical students. Acad Med. 2006;81(4):354-73. http:// dx.doi.org/10.1097/00001888-20060400000009 .

4. Henning MA, Krägeloh CU, Hawken SJ, Doherty I, Zhao Y, Shulruf B. Motivation to learn, quality of life and estimated academic achievement: medical students studying in New Zealand. Med Sci Educ. 2011;21(2):142-50. http://dx.doi.org/10. 1007/BF03341611.

5. Kusurkar RA, Croiset G, Galindo-Garre F, ten Cate O. Motivational profiles of medical students: association with study effort, academic performance and exhaustion. BMC Med Educ. 2013;13:87. http://dx.doi. org/10.1186/1472-6920-13-87.

6. Ten Cate TJ, Kusurkar RA, Williams GC. How self-determination theory can assist our understanding of the teaching and learning processes in medical education. AMEE guide No. 59. Med Teach. 2011;33(12): 961-73. http://dx.doi.org/10.3109/014215 9X.2011.595435.
7. Park J, Chung S, An H, Park S, Lee C, Kim SY, Lee JD, Kim KS. A structural model of stress, motivation, and academic performance in medical students. Psychiatry Investig. 2012;9(2):143-9. http://dx.doi.org/ 10.4306/pi.2012.9.2.143.

8. Yu TC, Wilson NC, Singh PP, Lemanu DP, Hawken SJ, Hill AG. Medical students-asteachers: a systematic review of peer-assisted teaching during medical school. Adv Med Educ Pract. 2011;2:157-72. http://dx.doi. org/10.2147/AMEP.S14383.

9. Moher D, Liberati A, Tetzlaff J, Altman DG. Preferred reporting items for systematic reviews and meta-analyses: the PRISMA statement. PLoS Med. 2009;6(7):e1000097-e. http://dx.doi.org/10. 1371/journal.pmed.1000097

10. Vallerand RJ, Pelletier LG, Blais MR, Hriere NM, Senecal C, Vallieres EF. The academic motivation scale: a measure of intrinsic, extrinsic, and amotivation in education. Educ Psychol Meas. 1992;52:1003-17. http://dx.doi.org/10.1177/001316449205200 4025 .

11. Del-Ben CM, Machado VF, Madisson MM, Resende TL, Valerio FP, Troncon LE. Relationship between academic performance and affective changes during the first year at medical school. Med Teach. 2013;35(5):404-10. http://dx.doi.org/10.310 9/0142159X.2013.769675.

12. Artino AR, La Rochelle JS, Durning SJ. Second-year medical students' motivational beliefs, emotions, and achievement. Med Educ. 2010;44(12):1203-12. http://dx.doi. org/10.1111/j.1365-2923.2010.03712.x.

13. Srivastava K, Raju MSVK, Saldanha D, Chaudhury S, Basannar D, Pawar AA, et al. Psychological well-being of medical students. Med J Armed Forces India. 2007;63(2):137-40. http://dx.doi.org/10. 1016/S0377-1237(07)80057-8. 
14. Henning MA, Hawken SJ, Krageloh C, Zhao Y, Doherty I. Asian medical students: quality of life and motivation to learn. Asia Pacific Education Review. 2011;12(3): 437-45. http://dx.doi.org/10.1007/s12564011-9148-y.

15. Artino AR, Hemmer PA, Durning SJ. Using self-regulated learning theory to understand the beliefs, emotions, and behaviors of struggling medical students. Acad Med. 2011;86(10 Suppl):S35-8. http://dx.doi.org/ 10.1097/ACM.0b013e31822a603d.

16. Huang CY, Liao HY, Chang SH. Social desirability and the clinical self-report inventory: methodological reconsideration. J Clin Psychol. 1998;54(4):517-28. http://dx. doi.org/10.1002/(SICI)1097-4679(199806) 54:4<517::AID-JCLP13>3.0.CO;2-I.

17. Dyrbye LN, Thomas MR, Eacker A, Harper W, Massie FS, Jr., Power DV, et al. Race, ethnicity, and medical student wellbeing in the United States. Arch Intern Med. 2007;167(19):2103-9. http://dx.doi. org/10.1001/archinte.167.19.2103.

18. Kusurkar RA, Croiset G, Mann KV, Custers $\mathrm{E}$, ten Cate $\mathrm{O}$. Have motivation theories guided the development and reform of medical education curricula? A review of the literature. Acad Med. 2012;87(6):735-43. http://dx.doi.org/10.1097/ACM.0b013e3182 53cc0e.
19. Sheldon KM, Krieger LS. Does legal education have undermining effects on law students? Evaluating changes in motivation, values, and well-being. Behav Sci Law. 2004;22(2):261-86. http://dx.doi. org/10.1002/bsl.582.

20. Ryan RM, Deci EL. Self-determination theory and the facilitation of intrinsic motivation, social development, and wellbeing. Am Psychol. 2000;55(1):68-78. http://dx.doi.org/10.1037/0003-066X.55. 1.68 .

21. Pintrich RR, DeGroot EV. Motivational and self-regulated learning components of classroom academic performance. J Educ Psychol. 1990;82:33-40. http://dx.doi.org/ 10.1037/0022-0663.82.1.33.

22. Wang P, Gao F. Mindful communication to address burnout, empathy, and attitudes. JAMA. 2010;303(4):330-1. http://dx.doi. org/10.1001/jama.2010.18.

23. Revzina L. Investigating social-desirability bias in self-reporting on motivational attitudes by adult students [dissertation]. Ann Arbor: University of San Francisco; 2008.

24. Kozma A, Stones MJ. Social desirability in measures of subjective well-being: a systematic evaluation. J Gerontol. 1987;42(1):56-9. http://dx.doi.org/10.1093/ geronj/42.1.56. 